\title{
Removing the barrier to the calculation of activation energies: Diffusion coefficients and reorientation times in liquid water
}

\author{
Zeke A. Piskulich, ${ }^{1,2}$ Oluwaseun O. Mesele, ${ }^{1}$ and Ward H. Thompson ${ }^{1,2, a)}$ \\ ${ }^{1}$ Department of Chemistry, University of Kansas, Lawrence, Kansas 66045, USA \\ ${ }^{2}$ Center for Environmentally Beneficial Catalysis, University of Kansas, Lawrence, Kansas 66047, USA
}

(Received 26 July 2017; accepted 14 September 2017; published online 3 October 2017)

\begin{abstract}
General approaches for directly calculating the temperature dependence of dynamical quantities from simulations at a single temperature are presented. The method is demonstrated for self-diffusion and $\mathrm{OH}$ reorientation in liquid water. For quantities which possess an activation energy, e.g., the diffusion coefficient and the reorientation time, the results from the direct calculation are in excellent agreement with those obtained from an Arrhenius plot. However, additional information is obtained, including the decomposition of the contributions to the activation energy. These results are discussed along with prospects for additional applications of the direct approach. Published by AIP Publishing. https://doi.org/10.1063/1.4997723
\end{abstract}

\section{INTRODUCTION}

Molecular dynamics (MD) simulations are a ubiquitous tool for understanding the dynamics of chemical systems. In particular, they can be directly connected with experimental measurements by, for example, using time correlation functions (TCFs) to obtain observables ranging from kinetic rate constants to vibrational spectra. Inevitably, the data generated in a MD simulation dwarf the information produced by such analyses. It is thus important to seek methods by which the trajectory data can be examined to take advantage of the significant information content that is generally discarded.

Reaction rate constants, transport coefficients, and other important dynamical time scales for molecular systems are frequently obtained through the calculation of TCFs. ${ }^{1-3}$ For example, the rate constant for a chemical reaction can be determined from the flux-side TCF, $C_{f s}(t)$, as ${ }^{4-7}$

$$
k=\lim _{t \rightarrow \text { long }} C_{f s}(t)=\lim _{t \rightarrow \text { long }}\left\langle F_{s}(0) \theta\left[s(t)-s^{\ddagger}\right]\right\rangle,
$$

where $s$ defines the dividing surface between reactants $\left(s<s^{\ddagger}\right)$ and products $\left(s>s^{\ddagger}\right), \theta(x)$ is the Heaviside step function, and $F_{s}(0)=\delta\left[s(0)-s^{\ddagger}\right] v_{s}(0)$ is the flux through the dividing surface with $v_{s}$ the velocity along $s$. The activation energy of the reaction,

$$
E_{a}=-\frac{d \ln k(T)}{d \beta},
$$

where $\beta=1 / k_{B} T$, is a quantity that is of significant interest due to the insight it provides into the effective barrier for the reaction. It is typically calculated by constructing an Arrhenius plot of $\ln k(T)$ versus $1 / T$ based on measurements (or calculations) at multiple temperatures. In the context of Eq. (1), however, $E_{a}$ is related to the temperature-dependence of a TCF such as $C_{f s}(t)$. Indeed, it has been shown by Dellago and Bolhuis ${ }^{8}$ that

a)Electronic mail: wthompson@ku.edu this perspective can be used to calculate $E_{a}$ directly from transition path sampling simulations at a single temperature, ${ }^{9-11}$ and a related approach has been demonstrated by Morita and co-workers for vibrational spectra. ${ }^{12,13}$ We have recently generalized this beyond transition path sampling and to other TCFs from which rate constants can be obtained, including those based on a quantum description. ${ }^{14}$

The measurement and calculation of activation energies by an Arrhenius analysis can raise significant issues due to the need to consider multiple temperatures. For example, many systems have a structure that is highly dependent on temperature, e.g., folded proteins, bilayer membranes or vesicles, and self-assembled structures. This can make the construction of an Arrhenius plot problematic by restricting the range of temperatures that can be considered. A similar issue arises even for bulk systems if one is interested in an activation energy at conditions that are near a phase transition. Yet it can be of significant interest to examine the activation energies of dynamical processes in the vicinity of these points of transformation.

It is also important to consider cases where quantities other than a rate constant are of interest, but for which an activation energy can still be measured and calculated. In this paper, we do just that by first showing a general expression for the temperature derivative of a TCF and then applying this result to two commonly considered dynamical properties: diffusion coefficients and reorientational time scales. The method is demonstrated by application to the relevant time correlation functions for these attributes in bulk liquid water. In addition, it is shown how deeper physical insight into these processes can be obtained than is possible from Arrhenius calculations.

\section{TIME CORRELATION FUNCTIONS (TCFS) AND ACTIVATION ENERGIES}

\section{A. General expressions}

A general time correlation function between two dynamical variables $A$ and $B$ in the canonical ensemble, 
$C_{A B}(t)=\langle A(0) B(t)\rangle$, can be written more explicitly as

$$
C_{A B}(t)=\frac{1}{Q} \operatorname{Tr}\left[e^{-\beta H} A(0) B(t)\right],
$$

where $Q$ is the partition function, $H$ is the Hamiltonian, and $\mathrm{Tr}$ indicates an integration over phase space. In this correlation function expression, only $Q$ and $e^{-\beta H}$ depend on the temperature. Thus, the derivative of $C_{A B}(t)$ with respect to $\beta$ can be straightforwardly evaluated as

$$
\begin{aligned}
\frac{\partial C_{A B}(t)}{\partial \beta}= & -\frac{1}{Q^{2}} \frac{d Q}{d \beta} \operatorname{Tr}\left[e^{-\beta H} A(0) B(t)\right] \\
& -\frac{1}{Q} \operatorname{Tr}\left[e^{-\beta H} H(0) A(0) B(t)\right] .
\end{aligned}
$$

Noting the definition of the average energy, $\langle H\rangle=-d \ln$ $Q / d \beta$, this gives

$$
\frac{\partial C_{A B}(t)}{\partial \beta}=-\langle\delta H(0) A(0) B(t)\rangle,
$$

where $\delta H(0)=H(0)-\langle H\rangle$ is the fluctuation in energy from its average value.

This shows that the temperature dependence of the TCF, and thus any associated time scales, can be determined by evaluating this simple time correlation function that is closely related to $C_{A B}(t)$ itself. Note that the interpretation of this result is intuitive as it relates the derivative to the (continuous) differences between $C_{A B}(t)$ when the system energy is initially greater than average $(\delta H(0)>0)$ and when it is initially less than average $(\delta H(0)<0)$. In this sense, Eq. (5) is an analytical derivative instead of the numerical derivative that is obtained from an Arrhenius analysis.

As noted above, an analogous result has already been obtained in the context of reactive flux and other TCFs that can be directly related to rate constants. ${ }^{8,14}$ However, there are many contexts in which time scale or transport coefficients are calculated from a TCF and possess an activation energy, or even simply a dependence on temperature, that is of physical interest. In the following, we show how the general result in Eq. (5) can be used to evaluate such activation energies for the examples of self-diffusion and $\mathrm{OH}$ reorientation in liquid water.

\section{B. Diffusion coefficients}

We consider, as a first example, the application of this formulation to the activation energy of diffusion. The diffusion coefficient, $D$, can be calculated from the mean-squareddisplacement (MSD) as

$$
M S D(t)=\left\langle|\vec{r}(t)-\vec{r}(0)|^{2}\right\rangle,
$$

which becomes linear at long times such that

$$
D=\lim _{t \rightarrow \text { long }} \frac{M S D(t)}{6 t},
$$

for motion in three dimensions. The activation energy associated with the diffusion coefficient is defined as

$$
E_{a}=-\frac{\partial \ln D}{\partial \beta}
$$

Then, taking the derivative of Eq. (7) with respect to $\beta$ and dividing by $D$ yields

$$
E_{a}=-\lim _{t \rightarrow \text { long }} \frac{1}{6 D t} \frac{\partial M S D(t)}{\partial \beta} .
$$

Using Eqs. (5)-(7), this can be written as

$$
\begin{aligned}
E_{a} & =\lim _{t \rightarrow \text { long }} \frac{\left\langle\delta H(0)|\vec{r}(t)-\vec{r}(0)|^{2}\right\rangle}{\left\langle|\vec{r}(t)-\vec{r}(0)|^{2}\right\rangle} \\
& \equiv \lim _{t \rightarrow \text { long }} \frac{M S D_{H}(t)}{M S D(t)},
\end{aligned}
$$

where $M S D_{H}(t)$, defined by the relation above, is the MSD weighted by the energy fluctuation. Note that the limit of the ratio of the functions equals the ratio of the limits as long as both are well defined and the limit of the denominator is not zero; each condition is met here. Thus, this ratio of TCFs should approach a constant value at longer times that is equal to the diffusion coefficient activation energy.

\section{Reorientation times}

The same approach can also be applied to reorientation dynamics. Reorientation times, denoted $\tau_{\ell}$, are typically calculated from the time decay of the $\ell$-th order reorientational correlation function,

$$
C_{\ell}(t)=\left\langle P_{\ell}[\vec{e}(0) \cdot \vec{e}(t)]\right\rangle
$$

Here $P_{\ell}$ is the $\ell$-th order Legendre polynomial and $\vec{e}$ is a unit vector pointing along some molecular axis, e.g., the $\mathrm{OH}$ bond in a water molecule. The $\ell=2 \mathrm{TCF}, C_{2}(t)$, is of particular interest as it can be directly measured by IR pump-probe anisotropy experiments, ${ }^{15-17}$ and an average rotational time defined as its integral can be obtained from NMR. ${ }^{16-20}$

In water and other hydrogen bonding (H-bonding) liquids, it has been shown that the $C_{2}(t)$ TCF is well described by a triexponential decay that distinguishes the time scales associated with reorientation due to inertial $\left(\tau_{\text {iner }}\right)$, librational $\left(\tau_{\text {lib }}\right)$, and $\mathrm{H}$-bond making and breaking $\left(\tau_{2}\right)$ dynamics. ${ }^{21}$ Taking this form for the general, $\ell$-th order case, the TCF can be written $\operatorname{as}^{22}$

$$
C_{\ell}(t)=\sum_{\alpha} A_{\alpha} e^{-t / \tau_{\alpha}},
$$

where $\alpha=$ iner, lib, and $\ell$ (corresponding to the three time scales), and $A_{\alpha}$ represent the amplitudes of the contributions of the three components to the overall reorientation dynamics. Applying the result in Eq. (5) to $C_{\ell}(t)$ gives

$$
-\frac{\partial C_{\ell}(t)}{\partial \beta}=C_{\ell, H}(t)=\left\langle\delta H(0) P_{\ell}[\vec{e}(0) \cdot \vec{e}(t)]\right\rangle,
$$

where $C_{\ell, H}(t)$ is the reorientational TCF weighted by the energy fluctuation. If the same derivative with respect to $\beta$ is taken in Eq. (12), an additional expression for $C_{\ell, H}(t)$ results, 


$$
C_{\ell, H}(t)=-\sum_{\alpha}\left[\frac{\partial A_{\alpha}}{\partial \beta}-\frac{\partial\left(1 / \tau_{\alpha}\right)}{\partial \beta} t A_{\alpha}\right] e^{-t / \tau_{\alpha}} .
$$

Then, Eq. (13) can be used to calculate $C_{\ell, H}(t)$ from MD simulations, while Eq. (14) gives the form to which it can be fit [constrained by $A_{\alpha}$ and $\tau_{\alpha}$ obtained by fitting $C_{\ell}(t)$ itself] to determine the temperature dependence of the amplitudes and time scales and, in some cases, the activation energy of the latter. In particular, an activation energy of one of the time scales can be obtained as

$$
E_{a, \alpha}=-\tau_{\alpha} \frac{\partial\left(1 / \tau_{\alpha}\right)}{\partial \beta}
$$

This again provides a simple method for calculating an activation energy from a single-temperature MD simulation.

\section{COMPUTATIONAL METHODS}

In principle, the energy fluctuation TCFs, $\operatorname{MSD}_{H}(t)$ and $C_{\ell, H}(t)$, and the associated activation energies can be calculated from a single $N V T$ MD simulation, as we have previously illustrated for H-bond exchange TCFs. ${ }^{14}$ However, there is naturally some effect due to the thermostat used to maintain the temperature that needs to be minimized. Here we adopt a different, nonequilibrium MD approach to illustrate the method that avoids any effect of the thermostat. Specifically, we sample initial conditions for short constant energy $(N V E)$ trajectories from a long $N V T$ MD simulation as illustrated in Fig. 1. Each $N V E$ trajectory has an initial energy, sampled from the canonical ensemble, that defines $\delta H(0)$ that weights the TCF giving $M S D(t)$ or $C_{2}(t)$.

For the results presented here, $2000 N V E$ trajectories of $20 \mathrm{ps}$ each were propagated starting from configurations and momenta sampled every $1 \mathrm{ps}$ from a $2 \mathrm{~ns} N V T$ trajectory following a $0.1 \mathrm{~ns}$ equilibration. For each trajectory, $M S D(t)$ and the reorientational TCF, $C_{2}(t)$, were calculated along with their energy fluctuation versions, $M S D_{H}(t)$ and $C_{2, H}(t)$. Uncertainties in the results are reported as $95 \%$ confidence intervals according to the Student's $t$-distribution based on block averaging with 8 blocks of 250 trajectories each.

A fully-periodic cubic simulation cell with a side length of $21.725311 \AA$ a was filled with 343 water molecules to give a density of $0.997 \mathrm{~g} / \mathrm{mol}$. The $\mathrm{H}_{2} \mathrm{O}$ molecules were modeled using the SPC/E force field. ${ }^{23}$ This model is a completely rigid, three-site model that treats intermolecular interactions as a combination of Lennard-Jones (LJ) and Coulombic

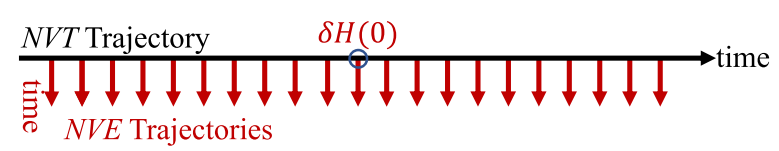

FIG. 1. A schematic diagram of the nonequilibrium MD simulation approach in which $N V E$ trajectories with different energies are initiated from a single NVT trajectory.

interactions. In this model, point charges are placed on each atom, while only oxygen atoms are treated as LJ sites. The Lorentz-Berthelot mixing rules are used to calculate intermolecular interactions between unlike atom types. ${ }^{24,25}$

The MD simulations were performed using the Large-Scale Atomic/Molecular Massively Parallel Simulator (LAMMPS) ${ }^{26,27}$ A simulation time step of $1.0 \mathrm{fs}$ was used, with configurations in the $N V E$ trajectories saved every $50 \mathrm{fs}$ for the calculation of the correlation functions. The SHAKE algorithm was used to hold the $\mathrm{OH}$ bonds and $\mathrm{H}-\mathrm{O}-\mathrm{H}$ angles rigid, ${ }^{28}$ with a tolerance of 0.0001 that specifies the relative error in the iterative solution. Intermolecular interactions were treated with a spherical cutoff of $10.5 \AA$ and long-range electrostatics were described with an Ewald summation with an accuracy parameter of 0.0001 (that specifies the root-meansquared error of the per-atom forces relative to a reference system). Canonical (NVT) simulations were performed using a Nosé-Hoover thermostat, ${ }^{29,30}$ with a thermostat damping parameter of $100 \mathrm{fs}$.

For comparison, activation energies were also calculated in the usual way using the Arrhenius equation from NVT trajectories at $T=285,298.15,315$, and $330 \mathrm{~K}$. Each trajectory was propagated for $4.5 \mathrm{~ns}$ with the first $0.5 \mathrm{~ns}$ used for equilibration. The trajectories were split into ten $0.4 \mathrm{~ns}$ blocks for block averaging to obtain $95 \%$ confidence intervals from the Student's $t$-distribution.

The results from the Arrhenius calculations are presented in Fig. 2. From linear fits of the Arrhenius plots, activation energies of $3.5 \pm 0.2$ and $3.5 \pm 0.1 \mathrm{kcal} / \mathrm{mol}$ are calculated for $D$ and $\tau_{2}$, respectively, in good agreement with previously reported values. ${ }^{33,34}$ These results will be used as a comparison for calculations using the energy fluctuation method described in Sec. II.

\section{RESULTS}

In this section, we apply the approaches described above to directly calculate the full temperature-dependence of TCFs
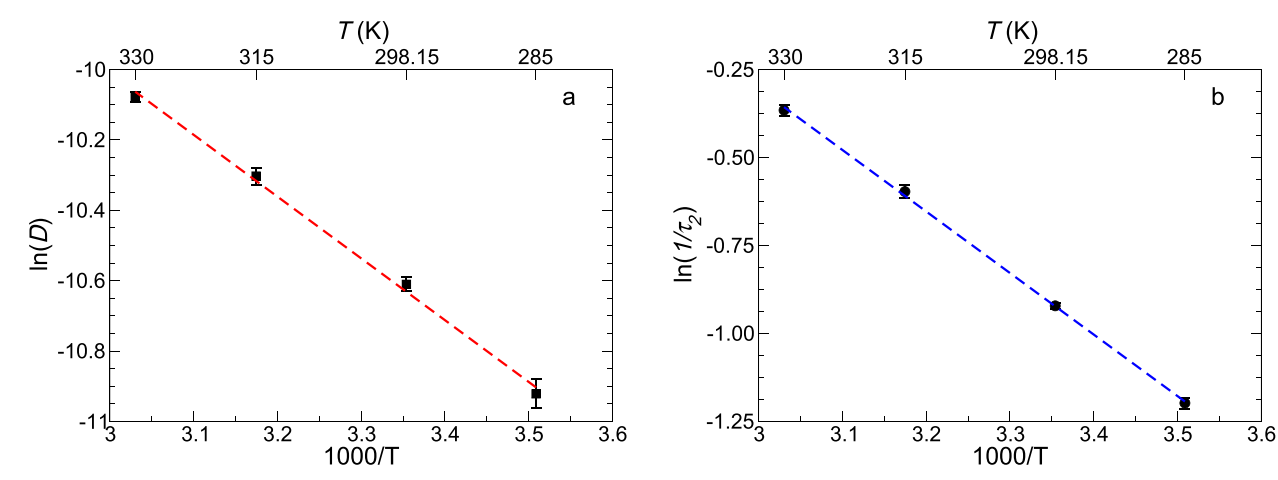

FIG. 2. Arrhenius plots for (a) $D$ and (b) $\tau_{2}$ are presented. Results from the MD simulations (filled black circles) are shown along with linear fits (dashed lines) based on the Arrhenius equation. 
and determine the activation energies associated with relevant time scales. We consider two examples involving bulk liquid water: self-diffusion and $\mathrm{OH}$-bond reorientation.

\section{A. Diffusion coefficient activation energy}

Using the nonequilibrium MD simulations described in Sec. III, we have calculated the mean-squared displacement, $M S D(t)$, and the corresponding energy fluctuation TCF, $M S D_{H}(t)$ for the oxygen atom of water at $298.15 \mathrm{~K}$. The results are presented in Fig. 3. A linear fit to $M S D(t)$ at longer times (between 2 and $20 \mathrm{ps}$ ) gives the diffusion coefficient as $D=2.5 \times 10^{-5} \mathrm{~cm}^{2} / \mathrm{s}$, in excellent agreement with reported values in the literature for the SPC/E water model. ${ }^{23,31,32}$ The time-dependence of $\operatorname{MSD}_{H}(t)$ is generally similar to $M S D(t)$ itself in that, following a short initial period, it is linear with time (with a slope of $5.3 \mathrm{kcal} / \mathrm{mol} \times \AA^{2} / \mathrm{ps}$ ).

As shown in Eq. (10), the activation energy for the diffusion coefficient can be calculated from the ratio $M S D_{H}(t) / M S D(t)$ at long times. This ratio is also shown in Fig. 3 as a function of time and does indeed reach a constant value for times longer than $\sim 12 \mathrm{ps}$. The value of the activation energy was obtained by fitting the ratio to a constant value for $t=15-20 \mathrm{ps}$, yielding $E_{a, D}=3.48 \pm 0.16 \mathrm{kcal} / \mathrm{mol}$. This result is in excellent agreement with the value of $3.5 \pm 0.2 \mathrm{kcal} / \mathrm{mol}$ obtained from the Arrhenius plot in Fig. 2. Note that an alternative method for calculating $E_{a, D}$ would be to use the ratio of the slopes obtained from the linear fits to the respective correlation functions; this yields $3.52 \mathrm{kcal} / \mathrm{mol}$ when fit over the time range $15-20 \mathrm{ps}$.

The calculation of diffusion activation energies in this manner provides an effective alternative to the usual Arrhenius method. Trajectories are required at only a single temperature and thus no choice needs to be made of the conditions for the simulations at other temperatures, e.g., whether to keep the same density (as we have done in our Arrhenius calculations) or modify the density to correspond to the experimental or simulation model result for each value of $T$. Additionally, as this approach calculates the temperature dependence of the

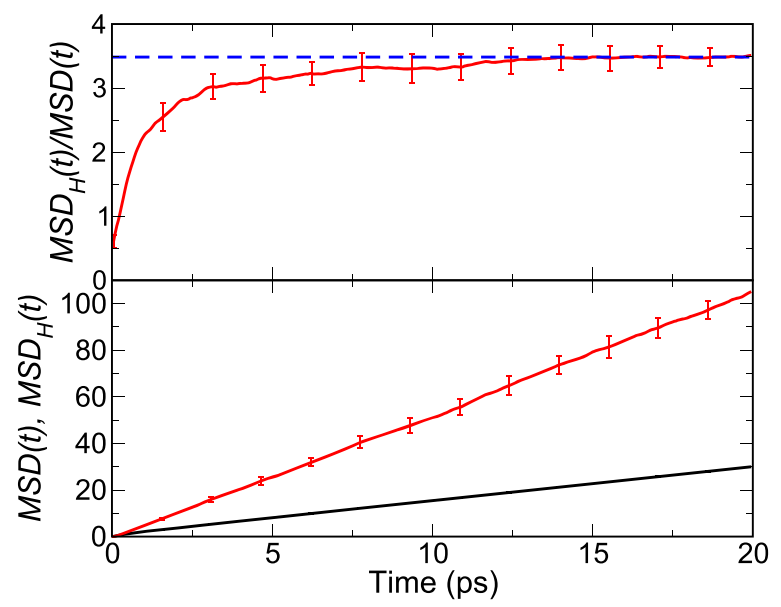

FIG. 3. (Bottom) The TCFs $M S D(t)$ (black line) and $M S D_{H}(t)$ (red line) are plotted versus time. (Top) The ratio $M S D_{H}(t) / M S D(t)$ (red line) is plotted as a function of time. A fit of this ratio between $t=15-20 \mathrm{ps}$ to a constant value is also shown (blue dashed line). Note: $M S D(t)$ is in units of $\AA^{2} / \mathrm{ps}$ and $M S D_{H}(t)$ in $\mathrm{kcal} / \mathrm{mol} \times \AA^{2} / \mathrm{ps}$. diffusion coefficient, it may be used for systems and conditions where the behavior is non-Arrhenius.

\section{B. Reorientation time activation energy}

The same nonequilibrium MD trajectories used to evaluate the diffusion coefficient were analyzed to calculate the reorientational correlation function, $C_{2}(t)$, and its temperature dependence via the energy fluctuation TCF, $C_{2, H}(t)$. These two TCFs are plotted as a function of time in Fig. 4 along with the tri-exponential fit to $C_{2}(t)$, Eq. (12), and the related fit to $C_{2, H}(t)$, Eq. (14). The former gives the three time scales for the reorientational dynamics as $25 \mathrm{fs}, 0.49 \mathrm{ps}$, and $2.6 \mathrm{ps}$, corresponding to inertial, librational, and $\mathrm{H}$-bonding breaking and making dynamics, respectively. These parameters are also used in the fit to $C_{2, H}(t)$ so that the fitting parameters are the derivatives with respect to $\beta$ of each of the amplitudes and time scales. Note that the fits to both $C_{2}(t)$ and $C_{2, H}(t)$ are in excellent agreement with the calculated TCFs.

A key focus of the analysis of the temperature dependence of the time scales is on the activation energy associated with the reorientational time $\tau_{2}$. The fit to $C_{2, H}(t)$ gives this as $E_{a, 2}=3.67 \pm 0.24 \mathrm{kcal} / \mathrm{mol}$. This agrees with the result of $3.5 \pm 0.1 \mathrm{kcal} / \mathrm{mol}$ obtained from the Arrhenius plot, Fig. 2(b), as well as prior calculations that also yielded $3.5 \mathrm{kcal} / \mathrm{mol} .^{33,34}$ It is notable that the activation energy for $\mathrm{OH}$ reorientation is similar to that obtained for self-diffusion. This is indicative of the common molecular origin of the two in the breaking and making of H-bonds. Namely, the exchange (or "jump") between two different $\mathrm{H}$-bond acceptors that is required for $\mathrm{OH}$ to reorient is also the key molecular event for diffusion of a water molecule.

It is important to note that because the derivatives with respect to $\beta$ are obtained from a simulation at a single temperature, they may or may not correspond to an activation energy. That is, it is not possible to determine from the derivative alone whether or not $\ln \left(1 / \tau_{\alpha}\right)$ depends linearly on $1 / T$. Often one has some prior knowledge, such as in the case of $D$ or $\tau_{2}$, that the property is activated. However, this is not true for the inertial and librational time scales, $\tau_{\text {iner }}$ and $\tau_{\text {lib }}$. An indication that they may not obey the Arrhenius behavior is

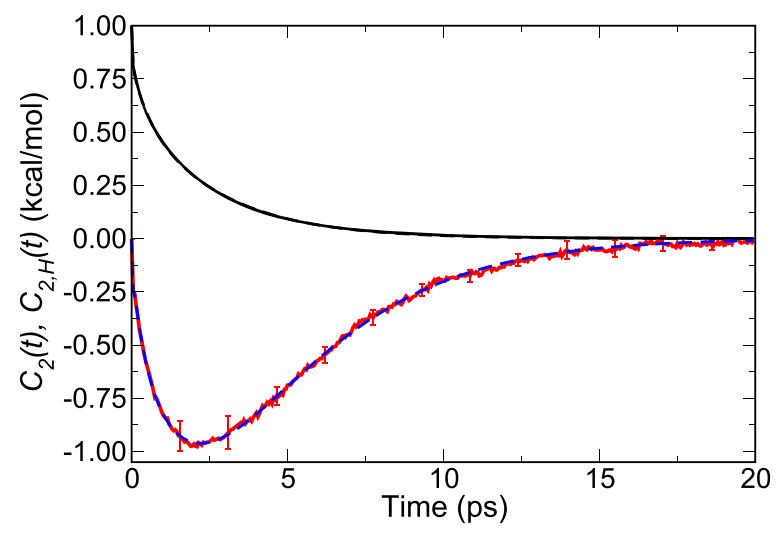

FIG. 4. The reorientational TCF $C_{2}(t)$ (solid black line) is shown as a function of time along with a tri-exponential fit (dashed black line) to Eq. (12). The TCF including the energy fluctuation, $C_{2, H}(t)$, is also shown (solid red line) along with the fit (dashed blue line) to Eq. (14). 
that our results give uncertainties that encompass zero activation energy: $E_{a, \text { iner }}=1.6 \pm 1.7 \mathrm{kcal} / \mathrm{mol}$ and $E_{a, l i b}=1.0 \pm 1.2$ $\mathrm{kcal} / \mathrm{mol}$. Indeed, plots of $1 / \tau_{\text {iner }}$ and $1 / \tau_{\text {lib }}$ versus $1 / T$ show that these time scales do not exhibit the Arrhenius behavior.

Additional information is available in the form of the amplitude derivatives. The fit to $C_{2, H}(t)$ gives $d A_{\text {iner }} / d \beta$ $=-0.11 \pm 0.09, d A_{l i b} / d \beta=-0.18 \pm 0.09$, and $d A_{2} / d \beta$ $=0.29 \pm 0.10$. These indicate that as temperature increases $(\beta$ decreases) the amplitudes of the inertial and librational components increase and those of the $\mathrm{H}$-bond making and breaking component decrease. This is consistent with linear fits to the amplitudes obtained from the simulations at different temperatures, which give $-0.08,-0.12$, and 0.20 for $A_{\text {iner }}, A_{\text {lib }}$, and $A_{2}$, respectively, for $T=285-315 \mathrm{~K}$. This is not the full temperature range we have simulated; however, the estimated derivatives change significantly (to $0.04,-0.12$, and 0.08 ) when $T=330 \mathrm{~K}$ is included in the fitting. This is a further indication that in the energy fluctuation TCFs we are obtaining local derivatives that can have distinct quantitative and qualitative differences from that obtained from multiple-temperature simulations that can depend on the temperature range considered.

\section{Energetic decomposition}

A key advantage of the method proposed in this work is the ability to decompose an activation energy or, more generally, a derivative with respect to temperature, into individual contributions due to each component of the energy. Specifically, we can note that the fluctuation in the energy that appears in $\operatorname{MSD}_{H}(t)$ and $C_{2, H}(t)$ can be written as

$$
\begin{aligned}
\delta H(0) & =\delta K E(0)+\delta V(0), \\
& =\delta K E(0)+\delta V_{L J}(0)+\delta V_{\text {Coul }}(0) .
\end{aligned}
$$

Here, $\delta K E(0)$ and $\delta V(0)$ are the fluctuations in the kinetic and potential energies, respectively. The second equality notes that the potential energy fluctuation can be further decomposed into the various types of interactions including this simplest example of the Lennard-Jones, $\delta V_{L J}$, and Coulombic, $\delta V_{C o u l}$, contributions to the water energy. Then, the activation energy can be likewise divided into such contributions as

$$
E_{a}=E_{a}^{K E}+E_{a}^{L J}+E_{a}^{C o u l},
$$

where $E_{a}^{K E}, E_{a}^{L J}$, and $E_{a}^{C o u l}$ are the components of the activation energy associated with fluctuations in the kinetic energy, LJ potential energy, and Coulombic potential energy, respectively. This approach builds on the pioneering studies of Tolman ${ }^{35}$ and Truhlar $^{36}$ to elucidate the physical interpretation of an activation energy.

In the case of diffusion, the component of the activation energy associated with the Coulombic interactions is, for example, then given by

$$
E_{a, D}^{\text {Coul }}=\frac{\left\langle\delta V_{\text {Coul }}(0)|\vec{r}(t)-\vec{r}(0)|^{2}\right\rangle}{\left\langle|\vec{r}(t)-\vec{r}(0)|^{2}\right\rangle}
$$

and similarly for $E_{a, D}^{K E}$ and $E_{a, D}^{L J}$. Note that this is not the only way to obtain insight into the origins of the activation energy and a particularly simple choice. In general, the energy fluctuation, $\delta H(0)$, can be divided up in any number of ways to gain insight into the nature of the activated process.

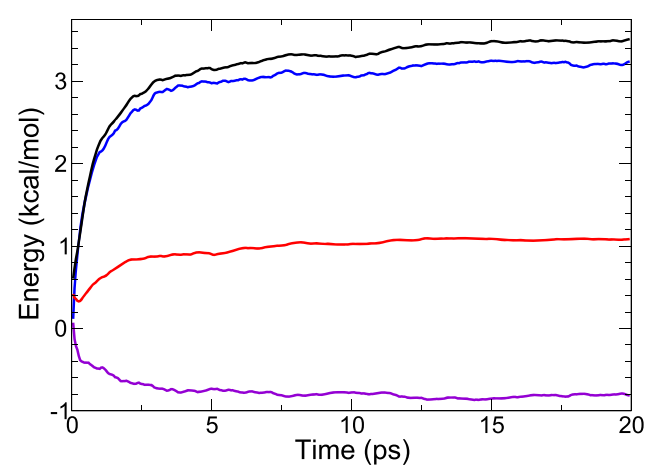

FIG. 5. The contributions to the diffusion TCF $M S D_{H}(t) / M S D(t)$ associated with the kinetic energy (red line), Lennard-Jones potential energy (violet line), and Coulombic potential energy (blue line) are plotted versus time along with the total $M S D_{H}(t) / M S D(t)$ (black line).

The resulting TCF for the Coulombic contribution to $M S D_{H}(t)$ given in Eq. (19) is presented as a function of time in Fig. 5 along with the LJ and kinetic energy results and the total $M S D_{H}(t)$. Each contribution to the activation energy can be obtained by fitting the constant value reached at longer times $(t=15-20 \mathrm{ps})$; this gives $1.1,-0.8$, and $3.2 \mathrm{kcal} / \mathrm{mol}$ for the kinetic energy, Lennard-Jones energy, and Coulombic energy, respectively. Thus, it is clear from the data that the dominant contribution to the activation energy of diffusion is the Coulombic interactions between water molecules. This is expected given the central role of $\mathrm{H}$-bonding in the mechanism of water diffusion. What is perhaps less obvious is the negative contribution from the Lennard-Jones interactions that are slightly more than that canceled by the kinetic energy component. These results point to the new insight that may be obtained by the present approach.

The same decomposition approach can be applied to the reorientational correlation function, $C_{2}(t)$. For example, $C_{2, H}(t)$ given in Eq. (13) can be written as the sum of

$$
C_{2, \text { Coul }}(t)=\left\langle\delta V_{\text {Coul }}(0) P_{\ell}[\vec{e}(0) \cdot \vec{e}(t)]\right\rangle
$$

and the analogous contributions $C_{2, K E}(t)$ and $C_{2, L J}(t)$. These three components are shown as a function of time, along with the total $C_{2, H}(t)$, in Fig. 6 . As with the diffusion constant, the dominant contribution to the activation energy is Coulombic

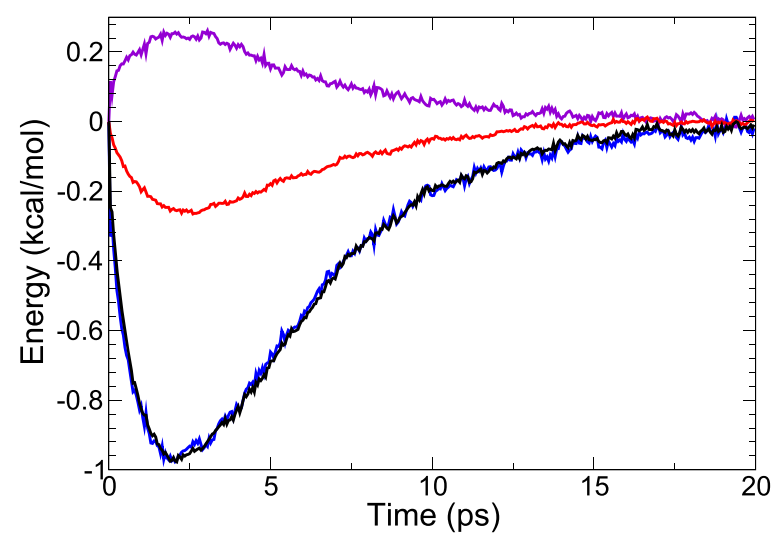

FIG. 6. The contributions to the reorientational TCF $C_{2, H}(t)$ associated with the kinetic energy (red line), Lennard-Jones potential energy (violet line), and Coulombic potential energy (blue line) are plotted versus time along with the total $C_{2, H}(t)$ (black line). 
interactions. In fact, there is essentially complete cancellation of the kinetic energy and LJ contributions such that the Coulombic component is nearly equal to the total $C_{2, H}(t)$ for all times. This is again an indication of the central role of $\mathrm{H}$ bond exchanges in $\mathrm{OH}$ reorientation in water, which has been extensively explored in the extended jump model of Laage and Hynes. ${ }^{33,37}$

\section{CONCLUSION}

This work demonstrates a general approach for evaluating the temperature-dependence of time correlation functions that can also yield the activation energy for transport coefficients or dynamical time scales. A key feature is that the activation energy is obtained from simulations at a single temperature. The method has been demonstrated for the self-diffusion and $\mathrm{OH}$ reorientation in bulk liquid water and gives activation energies in agreement with those obtained from standard Arrhenius calculations. The framework, however, is not limited to these examples and can be straightforwardly extended to other transport coefficients, dynamical time scales, or TCFs. Indeed, this approach gives the temperature derivative of a dynamical time scale at a given temperature and it does not require an assumption of the Arrhenius behavior.

This approach also provides additional insight into the origins of the activation energy. In particular, we have shown how the activation energy can be decomposed into components associated with the various contributions to the system energy, e.g., kinetic, Lennard-Jones, and Coulombic energies. For both diffusion and reorientation in bulk water, nearly the entire contribution to the activation energy arises from the Coulombic interactions, which is associated with the central role of hydrogen-bond dynamics in both processes. This kind of analysis should lead to a better understanding of the molecularlevel interactions that influence the activation energy.

Because the activation energy calculations do not require simulations at multiple temperatures, the present method may be particularly useful in cases where changing the temperature is problematic. For example, biological or self-assembled systems, such as lipid bilayers or reverse micelles, can often display dramatic changes in the structure, e.g., protein unfolding, with relatively small temperature changes. Similarly, for systems near a phase transition, the range of temperatures for which an Arrhenius analysis can be used is strongly constrained. However, the approach presented here permits the calculation of activation energies or, more generally, the derivative of full time correlation functions with respect to temperature even in such cases.

\section{ACKNOWLEDGMENTS}

The authors of this work thank the NSF-EPA Networks for Sustainable Materials Design and Synthesis (NSMDS) under
NSF Grant No. CHE-1339661 for funding. O.O.M. gratefully acknowledges support from a George E. Walrafen fellowship. The calculations were performed at the University of Kansas Center for Research Computing (CRC).

${ }^{1}$ R. Kubo, M. Toda, and N. Hashitsume, Statistical Physics II (SpringerVerlag, New York, 1978).

${ }^{2}$ D. Chandler, Introduction to Modern Statistical Mechanics (Oxford University Press, New York, 1987).

${ }^{3}$ R. Zwanzig, Nonequilibrium Modern Statistical Mechanics (Oxford University Press, New York, 2001).

${ }^{4}$ W. H. Miller, J. Chem. Phys. 61, 1823 (1974).

${ }^{5}$ W. H. Miller, S. D. Schwartz, and J. W. Tromp, J. Chem. Phys. 79, 4889 (1983).

${ }^{6}$ D. Chandler, J. Chem. Phys. 68, 2959 (1978).

${ }^{7}$ R. F. Grote and J. T. Hynes, J. Chem. Phys. 73, 2715 (1980).

${ }^{8}$ C. Dellago and P. G. Bolhuis, Mol. Simul. 30, 795 (2004).

${ }^{9}$ C. S. Lo, R. Radhakrishnan, and B. L. Trout, Catal. Today 105, 93 (2005).

${ }^{10}$ E. E. Borrero and C. Dellago, J. Chem. Phys. 133, 134112 (2010).

${ }^{11}$ C. Drechsel-Grau and M. Sprik, J. Chem. Phys. 136, 034506 (2012).

${ }^{12}$ S. Sakaguchi, T. Ishiyama, and A. Morita, J. Chem. Phys. 140, 144109 (2014).

${ }^{13}$ T. Joutsuka and A. Morita, J. Chem. Theory Comput. 12, 5026 (2016).

${ }^{14}$ O. O. Mesele and W. H. Thompson, J. Chem. Phys. 145, 134107 (2016).

${ }^{15}$ Y. S. Lin, P. A. Pieniazek, M. Yang, and J. L. Skinner, J. Chem. Phys. 132, 174505 (2010).

${ }^{16}$ H. J. Bakker and J. L. Skinner, Chem. Rev. 110, 1498 (2010).

${ }^{17}$ D. Laage, G. Stirnemann, F. Sterpone, R. Rey, and J. T. Hynes, Annu. Rev. Phys. Chem. 62, 395 (2011).

${ }^{18}$ D. W. G. Smith and J. G. Powles, Mol. Phys. 10, 451 (1966).

${ }^{19}$ M. D. Zeidler, Ber. Bunsengesellschaft Phys. Chem. 75, 769 (1971).

${ }^{20}$ J. Jonas, T. DeFries, and D. J. Wilbur, J. Chem. Phys. 65, 582 (1976).

${ }^{21}$ See, e.g., Ref. 17.

${ }^{22}$ It can be shown that the inertial dynamics give an initial Gaussian decay (see, e.g., D. A. McQuarrie, Statistical Mechanics, Harper-Collins, New York, 1976). However, it is more convenient to fit this component as an exponential decay like that associated with the librational and H-bond exchange dynamics. While this yields an approximate time scale for the inertial dynamics (which are not a focus of the present analysis), it does not affect the values obtained for $\tau_{l i b}$ or $\tau_{2}$, as we have verified by different fitting approaches.

${ }^{23}$ H. J. C. Berendsen, J. R. Grigera, and T. P. Straatsma, J. Phys. Chem. 91, 6269 (1987).

${ }^{24}$ H. Lorentz, Ann. Phys. 248, 127-136 (1881).

${ }^{25}$ D. Berthelot, Acad. Sci. 126, 1703-1855 (1898).

${ }^{26}$ S. J. Plimpton, J. Comput. Phys. 117, 1 (1995).

${ }^{27}$ See http://lammps.sandia.gov for the LAMMPS molecular dynamics package.

${ }^{28}$ G. Ciccotti and J. P. Ryckaert, Comput. Phys. Rep. 4, 346 (1986).

${ }^{29}$ S. Nosé, Mol. Phys. 52, 255 (1984).

${ }^{30}$ W. G. Hoover, Phys. Rev. A 31, 1695 (1985).

${ }^{31}$ We have not accounted for finite-size effects ${ }^{32}$ in calculating the diffusion constants or the corresponding activation energies in any of the approaches used. Thus, while the comparisons are between consistent descriptions, the numerical results may be modified by such corrections.

${ }^{32}$ I.-C. Yeh and G. Hummer, J. Phys. Chem. B 108, 15873 (2004).

${ }^{33}$ D. Laage and J. T. Hynes, J. Phys. Chem. B 112, 14230 (2008).

${ }^{34}$ A. A. Vartia, K. R. Mitchell-Koch, G. Stirnemann, D. Laage, and W. H. Thompson, J. Phys. Chem. B 115, 12173 (2011).

${ }^{35}$ R. C. Tolman, J. Am. Chem. Soc. 42, 2506 (1920).

${ }^{36}$ D. G. Truhlar, J. Chem. Educ. 55, 309 (1978).

${ }^{37}$ D. Laage and J. T. Hynes, Science 311, 832 (2006). 